\title{
Analytical Solution to Normal Forms of Hamiltonian Systems
}

\author{
Ali Allahem
}

Department of Mathematics, College of Sciences, Qassim University, P.O. Box 6666, Buraydah 51452, Saudi Arabia; a.allahem@qu.edu.sa

Received: 10 June 2017; Accepted: 26 July 2017; Published: 27 July 2017

\begin{abstract}
The idea of the normalisation of the Hamiltonian system is to simplify the system by transforming Hamiltonian canonically to an easy system. It is under symplectic conditions that the Hamiltonian is preserved under a specific transformation-the so-called Lie transformation. In this review, we will show how to compute the normal form for the Hamiltonian, including computing the general function analytically. A clear example has been studied to illustrate the normal form theory, which can be used as a guide for arbitrary problems.
\end{abstract}

Keywords: Hamiltonian; normal forms; generating function; Lie transformation; canonical transformation

\section{Introduction}

A Hamiltonian system is a dynamical system that satisfies

$$
\dot{q}=\frac{\partial H}{\partial p}, \quad \dot{p}=-\frac{\partial H}{\partial q}
$$

ordinary differential equations (ODEs). Here, $q \in R^{n}$ represents the coordinates of the configuration variable (positions) and their canonically conjugate momenta $p \in R^{n}$. The function $H=H(q, p, t)$ is called the Hamiltonian of System (1) with $n$ degrees of freedom ( $n$ dof). We may write the Hamiltonian system

$$
\dot{\mathbf{y}}=\mathbf{J} \nabla H(\mathbf{y}, t)
$$

where $\mathbf{J}$ is the $2 n \times 2 n$ Poisson matrix $\mathbf{J}=\left[\begin{array}{cc}0 & I_{n} \\ -I_{n} & 0\end{array}\right]$ and $\mathbf{y}=(q, p)$. Furthermore, the Hamiltonian $H$ possesses an equilibrium $y^{0}$ (i.e., $\frac{\partial H}{\partial y}\left(y^{0}\right)=0$ ) at the origin in $R^{2 n}$. If not, we make the shift $y=\hat{y}+y^{0}$ zero. For many dynamical systems, the Hamiltonian $H$ represents the energy in the system. Furthermore, the Hamiltonian $H$ will be in the form $H(q, p)=T+V$, where $T$ is the kinetic energy and $V$ is the potential energy of the system and is a function of $q$ alone. The energy is constant, if a Hamiltonian does not depend explicitly on the time $t$ :

$$
\frac{d H}{d t}=\frac{\partial H}{\partial q} \frac{d q}{d t}+\frac{\partial H}{\partial p} \frac{d p}{d t}=0
$$

by (1). Hence, $H(q(t), p(t))=H(q(0), p(0))=E$. This is called conservation of energy. Where the Hamiltonian depends on time $H(q, p, t)$, the energy is not conserved [1-3].

\section{Methodology}

Here, we will provide a brief description of the normal form of the Hamiltonian system process using the Lie transform [4-8]. We are going to transform a given Hamiltonian $H=H(q, p)$ 
into another Hamiltonian $K=K(Q, P)$ which is simpler by means of a canonical transformation $Q=Q(q, p, t)$ and $P=P(q, p, t)$.

If we define the Hamiltonian function $H(q, p, t)$ and transformed Hamiltonian function $K(Q, P, t)$ such that

$$
\begin{aligned}
& \dot{q}=\frac{\partial H}{\partial p}, \quad \dot{p}=-\frac{\partial H}{\partial q}, \\
& \dot{Q}=\frac{\partial K}{\partial P}, \quad \dot{P}=-\frac{\partial K}{\partial Q^{\prime}}
\end{aligned}
$$

then the coordinate transformation $(q, p) \rightarrow(Q, P)$, such that $Q=Q(q, p, t)$ and $P=P(q, p, t)$, is the so-called canonical transformation [9]. However, in our example, we use the exterior product to verify whether the transformations are canonical or not by using its properties [10,11]. In detail, if $A(v, w)$ denotes the area of the parallelogram determined by the pair of vectors $v$ and $w$ then $A$ has the following properties:

- $A(v, v)=0$.

- $\quad A(v, w)=-A(w, v)$.

Apply these two properties in the transformation $(q, p) \rightarrow(Q, P)$ such that

$$
\omega^{2}=d q_{1} \wedge d p_{1}+d q_{2} \wedge d p_{2}+\ldots
$$

and

$$
\omega^{2}=d Q_{1} \wedge d P_{1}+d Q_{2} \wedge d P_{2}+\ldots
$$

if $\sum_{i=1}^{n} d q_{i} \wedge d p_{i}=\sum_{i=1}^{n} d Q_{i} \wedge d P_{i}$, then the transformations are canonical.

Lie transformation provides a symplectic change of the variable that depends on a small parameter as the general solution of the Hamiltonian system [4,5]. In detail, the general solution $\mathcal{X}(\mathbf{y}, \epsilon)$ defines a canonical transformation such that $\mathbf{x}=\mathcal{X}(\mathbf{y})$ with the inverse $\mathbf{y}=\mathcal{Y}(\mathbf{x})$, and hence $\mathcal{X}(\mathcal{Y}(\mathbf{x}))=\mathbf{x}$, where the time $\epsilon$ maps the flow of the Hamiltonian system. It is defined by the generation function $W$. $[4,5]$ We will set time $\epsilon=1$ in order to make our transformations canonical. We then have

$$
\mathbf{x}=\mathcal{X}(\mathbf{y}):=\mathcal{X}(\mathbf{y}, \epsilon=1)=\left.\mathcal{X}\right|_{\epsilon=1}(\mathbf{y})
$$

\subsection{Generating Function}

The generating function $W(\mathbf{y}, \epsilon)$ is an auxiliary non-autonomous Hamiltonian depending on the parameter $\epsilon$, and the coordinates $\mathbf{y}=(q, p)=\left(q_{1}, . ., q_{n}, p_{1}, \ldots, p_{n}\right)$. The Hamiltonian system associated with the generating function is given by

$$
\begin{gathered}
\frac{d q}{d \epsilon}=\frac{\partial W(\mathbf{y}, \epsilon)}{\partial p}, \\
\frac{d p}{d \epsilon}=\frac{-\partial W(\mathbf{y}, \epsilon)}{\partial q} .
\end{gathered}
$$

The general solution is written as $\mathcal{X}:(\mathbf{y}, \epsilon) \mapsto \mathcal{X}(\mathbf{y}, \epsilon)$. For example, the solution curve through a particular point $y^{*}$ can be written as

$$
\mathcal{X}_{y^{*}}: \epsilon \mapsto \mathcal{X}\left(y^{*} ; \epsilon\right)
$$

with initial condition $\mathbf{x}=(\mathbf{y}, 0)=\left.\mathcal{X}(\mathbf{y}, \epsilon)\right|_{\epsilon=0}=\mathbf{y}$. 
To make sure that the transformation between the original Hamiltonian and the new one is valid, we resort to an indirect generating function approach that can be derived from an action principle of the form

$$
S_{q p}=\int_{t_{0}}^{t_{1}}[p \dot{q}-H(q, p, t)] d t
$$

Let us consider the independent variations $\delta q, \delta p$ and ask the action to be minimized with respect to these variations:

$$
\begin{aligned}
0=\delta S_{q p} & =\int_{t_{0}}^{t_{1}}\left[p \delta \dot{q}+\dot{q} \delta p-\frac{\partial H}{\partial q} \delta q-\frac{\partial H}{\partial p} \delta p\right] d t \\
& =\int_{t_{0}}^{t_{1}}\left[\left(-\dot{p}-\frac{\partial H}{\partial q}\right) \delta q+\left(\dot{q}-\frac{\partial H}{\partial p}\right) \delta p\right] d t+\left.[p \delta q]\right|_{t_{0}} ^{t_{1}}
\end{aligned}
$$

Similarly, we calculate the variation of the action in $P$ and $Q$ :

$$
\delta S_{Q P}=\int_{t_{0}}^{t_{1}}\left[\left(-\dot{P}-\frac{\partial K}{\partial Q}\right) \delta Q+\left(\dot{Q}-\frac{\partial K}{\partial P}\right) \delta P\right] d t+\left.[P \delta Q]\right|_{t_{0}} ^{t_{1}} .
$$

We need to show that the integral term vanishes for any variations $\delta Q, \delta P$. Furthermore, the solution to the action principle is unchanged if $S_{q p}-S_{Q P}=\left.W\right|_{t_{0}} ^{t_{1}}$, where $W=W(q, t)$ is a function of coordinates and time. If we use this condition of the action principle and keep both $q$ and $p$ fixed at the initial and final times, then $W$ is a function of coordinates and momenta $W=W(q, p, t)$. To summarize, we have demonstrated a sufficient condition for the transformation $(q, p) \rightarrow(Q, P)$ to be a canonical transformation, if there is a function $W(q, Q, t)$ such that

$$
p=\frac{\partial W}{\partial q} \quad P=-\frac{\partial W}{\partial Q}, \quad K=H+\frac{\partial W}{\partial t},
$$

where $W$ is called a generating function. Note that if the system does not depend explicitly on time $t$ then the new Hamiltonian function is the same as the old Hamiltonian function. There are four types of generating functions. All have old coordinates or old momenta and new coordinates or new momenta, respectively. Moreover, the generating function can be determined with respect to the normal forms. It has a different process to calculate. In the next section, we will explain the normal form of a Hamiltonian and then provide an example to clarify the theoretical part.

\subsection{Normal Form}

We write the coordinate change as

$$
\mathbf{x}=\mathcal{X}(\mathbf{y}), \quad \mathbf{y}=\mathcal{Y}(\mathbf{x})
$$

with $\mathcal{X}(\mathcal{Y}(\mathbf{x}))=\mathbf{x}$, where $\mathbf{x}=\left(x_{1}, \ldots, x_{2 n}\right)=\left(q_{1}, \ldots, q_{n}, p_{1}, \ldots, p_{n}\right)$ and $\mathbf{y}=\left(y_{1}, \ldots, y_{2 n}\right)=$ $\left(Q_{1}, \ldots, Q_{n}, P_{1}, \ldots, P_{n}\right)$. Suppose the Hamiltonian function $H$ depends on a parameter $\epsilon$ :

$$
H=\sum_{i=0}^{\infty} \frac{\epsilon^{i}}{i !} H_{i}(\mathbf{x}, \epsilon)
$$

where $x_{i}, 1 \geq i \geq n$ refers to the coordinates and $x_{i}, n+1 \geq i \geq 2 n$ refers to their conjugate momenta. The transformed Hamiltonian $K$ also depends on a parameter $\epsilon$ :

$$
K=\sum_{i=0}^{\infty} \frac{\epsilon^{i}}{i !} K_{i}(\mathbf{y}, \epsilon) \equiv \sum_{i=0}^{\infty} \frac{\epsilon^{i}}{i !} H_{0}^{(i)}(\mathbf{y}, \epsilon)
$$

where $x_{i}, 1 \geq i \geq n$ refers to the transformed coordinates and $x_{i}, n+1 \geq i \geq 2 n$ refers to their conjugate momenta. 
Lie transformation can be achieved by using the solution to another Hamiltonian system defined by the generating function

$$
W=\sum_{i=0}^{\infty} \frac{\epsilon^{i}}{i !} W_{i+1}(\mathbf{y}, \epsilon), \quad \text { where } \quad W_{i+1}(\mathbf{y})=\left(\frac{\partial^{i}}{\partial \epsilon^{i}} W(\mathbf{y}, \epsilon)\right),
$$

following the recursion formula

$$
H_{i}^{(j)}=H_{i+1}^{(j-1)}+\sum_{k=0}^{i}\left(\begin{array}{l}
i \\
k
\end{array}\right)\left\{H_{i-k}^{(j-1)}, W_{k+1}\right\}
$$

with $i \geq 0, j \geq 1$, and hence, $H_{(0)}^{i}=H_{i}$. The operator $\{\cdot, \cdot\}$ is the so-called Poisson bracket of two scalar fields: given $A, B: \mathrm{R}^{2 n} \rightarrow \mathrm{R}$ and is defined as the quantity

$$
\{A, B\}=\sum_{i=1}^{n} \frac{\partial A}{\partial x_{i}} \frac{\partial B}{\partial x_{n+i}}-\frac{\partial A}{\partial x_{n+i}} \frac{\partial B}{\partial x_{i}}
$$

where $q, p$ are coordinates and momenta, respectively [12].

Note that $W(\mathbf{x}, \epsilon)$ is conserved under the transformation and can be written as $W(\mathbf{x}, \epsilon)=W(\mathbf{y}, \epsilon)$. means of

We express the original Hamiltonian $(H)$ in terms of the new variable $(Q, P)$ as $K=K(Q, P, \epsilon)$ by

$$
K(Q, P, \epsilon) \equiv H(\underbrace{q(Q, P, \epsilon), p(Q, P, \epsilon)}_{\mathcal{X}(\mathbf{y}, \epsilon)}, \epsilon),
$$

where $\mathcal{X}(\mathbf{y}, \epsilon)=\sum_{i=0}^{\infty} \frac{\epsilon^{i}}{i !} \mathcal{X}_{i}(\mathbf{y}, \epsilon)$. The coordinate change will be a near-identity map, which means that $\mathcal{X}_{0}(\mathbf{y})=\mathbf{y}$ and thus,

$$
\mathcal{X}(\mathbf{y}, \epsilon)=\mathbf{y}+\sum_{i=1}^{\infty} \frac{\epsilon^{i}}{i !} \mathcal{X}_{i}(\mathbf{y}, \epsilon)
$$

A similar formula can be used to see the change of coordinates back to the old ones, which is

$$
\mathcal{Y}(\mathbf{x}, \epsilon)=\mathbf{x}+\sum_{i=1}^{\infty} \frac{\epsilon^{i}}{i !} \mathcal{Y}_{i}(\mathbf{x}, \epsilon)
$$

Here, we define the quantities $L_{j}^{i}$ as the relation between the coefficients of the various series that are expressed in terms of intermediate quantities $L_{j}^{i}$ with $0 \leq j, i \leq n$ and $j+i=n$. These quantities can compute the transformed Hamiltonian $K_{n}$ from the original Hamiltonian $H_{n}$ and other quantities are computed by a chain of relations:

$$
H_{n} \equiv L_{0}^{n} \rightarrow L_{1}^{n-1} \rightarrow \ldots \rightarrow L_{n-1}^{1} \rightarrow L_{n}^{0} \equiv K_{n} .
$$

The following recursion formula relates the terms $K$ with those in $H$ and $W$ by quantities $L_{j}^{i}$

$$
L_{j}^{i}=L_{j+1}^{i-1}+\sum_{k=0}^{j}\left(\begin{array}{l}
j \\
k
\end{array}\right)\left\{L_{j-k^{\prime}}^{i-1} W_{k+1}\right\}
$$

with $j \geq 0$ and $i \geq 1[9,13,14]$.

The last Equation (5) has the binomial coefficient $\left(\begin{array}{l}j \\ k\end{array}\right)=\frac{j !}{k !(j-k) !}$. Note that the calculation of $L_{0}^{0}$ makes the first term $H_{0}$ and $K_{0}$ equal because $W$ is a near identity transformation, and hence, the transformation is generated [12]. In addition, the first term in the expansion for $W$ starts with $W_{1}$. 
The normal form process can be stopped at any existing order. The Lie triangle summarizes the recursion process $[4,5]$. Thus, the process and Lie triangle are as follows:

$$
\begin{aligned}
K_{0}: \quad K_{0} \equiv L_{0}^{0} & =H_{0} \\
K_{1}: \quad K_{1} \equiv L_{1}^{0} & =L_{1}^{0}+\left\{W_{3}, L_{0}^{0}\right\} \\
& =H_{1}+\left\{W_{1}, H_{0}\right\} \\
K_{2}: \quad K_{2} \equiv L_{2}^{0} & =L_{1}^{1}+\left\{W_{1}, L_{1}^{0}\right\} \\
& =L_{2}^{0}+\left\{W_{1}, L_{1}^{0}\right\}+\left\{W_{2}, L_{0}^{0}\right\}+\left\{W_{1}, L_{1}^{0}\right\} \\
& =H_{2}+\left\{W_{1}, H_{1}\right\}+\left\{W_{2}, H_{0}\right\}+\left\{H_{1}+\left\{W_{1}, H_{0}\right\}, W_{1}\right\} \\
& =H_{2}+\left\{W_{1}, H_{1}\right\}+\left\{W_{2}, H_{0}\right\}+\left\{W_{1}, H_{1}\right\}+\left\{\left\{W_{1}, H_{0}\right\}, W_{1}\right\} \\
& =H_{2}+2\left\{W_{1}, H_{1}\right\}+\left\{W_{2}, H_{0}\right\}+\left\{\left\{W_{1}, H_{0}\right\}, W_{1}\right\},
\end{aligned}
$$

where $K_{i}$ is the transformed Hamiltonian and

$$
\begin{array}{cccccccccc}
L_{0}^{0} & & & & & & & & \\
\downarrow & \searrow & & & & & & \\
L_{1}^{0} & \rightarrow & L_{0}^{1} & & & & & & \\
\downarrow & & \downarrow & \searrow & & & & & \\
L_{2}^{0} & \rightarrow & L_{1}^{1} & \rightarrow & L_{0}^{2} & & & & \\
\downarrow & & \downarrow & & \downarrow & \searrow & & & \\
L_{3}^{0} & \rightarrow & L_{2}^{1} & \rightarrow & L_{1}^{2} & \rightarrow & L_{0}^{3} & \\
\vdots & \ddots & \vdots & \ddots & \vdots & \ddots & \vdots & \ddots,
\end{array}
$$

respectively [9]. By using Lie transformations to compute the normal form, the transformed Hamiltonian $K$ is defined as

$$
K=K_{0}+K_{1}+\ldots
$$

In general, $K$ is in normal form, where $K_{n}$ is the polynomial of the degree $n+2$. In addition, for any smooth function $f$, then

$$
\left\{f, K_{i}\right\}=0 \text {, with } i=0,1, \ldots .
$$

This is the so-called normal form with respect to a given function. However, the same property can be applied on its own quadratic terms, namely $K_{2}$ such that $\left\{K_{0}, K_{i}\right\}=0$. Thus, the normal form of quadratic terms $K_{0}$ has the form

$$
K_{0}(\mathbf{y})=\sum_{j=1}^{n} \lambda_{j}\left(q_{j} p_{j}\right)
$$

where $q_{j}$ and $p_{j}$ are configuration space coordinates and their conjugate momenta, respectively. Additionally, the coefficients of products $q_{j} p_{j}$ are given by the vector $\lambda=\left(\lambda_{1}, \ldots, \lambda_{n}\right) \in \mathbf{C}^{n}$.

To sum up, assume that we have the Hamiltonian such that

$$
H=H_{0}+H_{1}+\ldots,
$$

with $H_{n}$ is the polynomial of degree $n+2$ and its coordinates denoted by $\mathbf{x}=(q, p)$. The aim of normalization is to find the easiest change of coordinates

$$
\mathbf{x}=\mathcal{X}(\mathbf{y}), \quad(\text { canonical })
$$

with the inverse

$$
\mathbf{y}=\mathcal{Y}(\mathbf{x})
$$

through the generating function $W$, such that the function $H$ expressed in terms of $\mathbf{y}$ by means of

$$
H(\mathcal{X}(\mathbf{y}))=K(\mathbf{y})
$$


with $K=K_{0}+K_{1}+\ldots$ results in a transformed Hamiltonian $K$, that is in the normal form through the degree $n+2$.

The above method was first proposed by Deprit [5]. Here, we have followed the presentation style of [9].

\subsection{Computing a Generating Function $W$}

In more detail, we provide an example to show how to find generating functions $W_{3}$ and $W_{4}$ of the degree three and four in formal norm [15].

Consider $H$ to be a Hamiltonian of ( $n$ dof). Let us expand $H$ in power series such that

$$
H(q, p)=H_{2}(q, p)+\epsilon H_{3}(q, p)+\ldots,
$$

where $H_{n}(q, p)$ is a homogeneous polynomial of degree $n$ in the variables $(q, p)$. The aim is to perform transformations canonically to make the expansion simple. We will perform all series manipulations formally, and set $\epsilon=1$ afterwards.

As we have (7), then

$$
\begin{aligned}
K & =e^{\epsilon\left\{W_{3}, \cdot\right\}} H, \\
& =\left[I+\epsilon\left\{W_{3}, \cdot\right\}+\frac{\epsilon^{2}}{2}\left[W_{3},\left\{W_{3}, \cdot\right\}\right\}\right] H, \\
& =H_{2}+\epsilon\left(H_{3}+\left\{W_{3}, H_{2}\right\}\right)+\mathcal{O}\left(\epsilon^{2}\right),
\end{aligned}
$$

where $K$ is the transformed Hamiltonian. It is easy to see that the monomials of degree three of $K$ can be obtained using generating function $W_{3}$ by

$$
\bar{H}_{3}=H_{3}+\left\{W_{3}, H_{2}\right\}
$$

We choose the coefficients of $W_{3}$ such that $\bar{H}_{3}$ is zero.

Note that if we assume $\mathbf{x}=\left(x_{1}, x_{2}, \ldots, x_{n}\right)$ and $\mathbf{k}=\left(k_{1}, \ldots, k_{n}\right) \in N^{n}$, and we define

$$
\mathbf{x}^{\mathbf{k}}=x_{1}^{k_{1}} \ldots x_{n}^{k_{n}} \quad \text { and } \quad|k|=k_{1}+\ldots+k_{n}
$$

hence $H_{3}$ and $W_{3}$ can be written as

$$
\begin{aligned}
& H_{3}(q, p)=\sum_{\left|k_{q}\right|+\left|k_{p}\right|=3} h_{k_{q} k_{p}} q^{k_{q}} p^{k_{p}}, \\
& W_{3}(q, p)=\sum_{\left|k_{q}\right|+\left|k_{p}\right|=3} w_{k_{q} k_{p}} q^{k_{q}} p^{k_{p}} .
\end{aligned}
$$

We determine the coefficients such that $\left\{W_{3}, H_{2}\right\}=-H_{3}$. Note that $\left\{H_{2}, \cdot\right\}$ is a linear operator and takes the diagonal form, due to

$$
\left\{H_{2}, q^{k_{q}} p^{k_{p}}\right\}=i\left\langle k_{p}-k_{q}, \omega\right\rangle q^{k_{q}} p^{k_{p}} .
$$

Hence, it is easy to find $W_{3}$ such that

$$
W_{3}(q, p)=\sum_{\left|k_{q}\right|+\left|k_{p}\right|=3}=\frac{-h_{k_{q} k_{p}}}{i\left\langle k_{p}-k_{q}, \omega\right\rangle} q^{k_{q}} p^{k_{p}} .
$$

However, $\langle k, \omega\rangle$ do not vanish for any $k \in Z-\{0\}$. If the components of frequency vector $\boldsymbol{\omega}=\left(\omega_{1}, \omega_{2}, \ldots, \omega_{l}\right)$ are linearly independent and $\left|k_{q}\right|+\left|k_{p}\right|=3$, then this condition is satisfied. Once $W_{3}$ has been calculated, we can compute the new coordinates as a function of the old ones and vice versa $[9,12,13]$. 
We rewrite the transformed Hamiltonian as function of $H$ such that

$$
H(q, p)=H_{2}(q, p)+H_{4}(q, p)+H_{5}(q, p)+\ldots
$$

The following step is calculating the generating function $W_{4}$ to get rid of the monomials of degree four from $H$. In general, this cannot be applied because $\left\{H_{2}, \cdot\right\}$ has some zero eigenvalues:

$$
\left\{H_{2}, q^{k} p^{k}\right\}=0 .
$$

Thus, we can only solve the equation $\left\{W_{4}, H_{2}\right\}=-H_{4}$, if the form of $H_{4}$ is $q^{k_{q}} p^{k_{p}}$, with $k_{q} \neq k_{p}$ :

$$
W_{4}(q, p)=\sum_{\left|k_{q}\right|+\left|k_{p}\right|=4}=\frac{-h_{k_{q} k_{p}}}{i\left\langle k_{p}-k_{q}, \omega\right\rangle} q^{k_{q}} p^{k_{p}}
$$

The presented method is formal without looking at the convergence of the variables. There are many applications presented in the series divergence. The important part of the method process is the first orders of the transformed system, which provide interesting information due to the linear approximation around the equilibrium. The process can be studied up to any existing order $(\epsilon)$ for a good approximation [16,17]. In other words, the first order terms consist of useful information to reduce the transformed system without being affected by the divergent character, where the general perturbation theorem takes place.

To summarize, once the generating function $W$ is calculated, we can derive the new coordinates as functions of the old ones and vice versa. Additionally, the generating function $W$ and the calculations of Poisson brackets can be used to see the coordinates changing back to the old ones without any additional calculations. There are some interesting examples in physics and engineering for the idea of Hamiltonians normal forms and generating functions which can be found in the book by Sanders and Verhulst [18]. An example follows the theoretical part for clarification.

\section{Example}

Let us consider the Hamiltonian function that is defined as

$$
H=\frac{1}{2}\left(p_{x}^{2}+p_{y}^{2}\right)-\frac{1}{2} \omega_{b}^{2} x^{2}+\frac{1}{2} \omega_{y}^{2} y^{2}+a x^{3}+b x y^{2} .
$$

Firstly, we rescale $p_{x}, p_{y}, x$ and $y$ such that

$$
q_{1}=\frac{x}{\omega_{b}}, \quad q_{2}=\frac{y}{\omega_{y}}, \quad p_{1}=\omega_{b} p_{x}, \quad p_{2}=\omega_{y} p_{y}
$$

This rescaling is canonical and easy to check. It is also easy to see that

$$
d q_{1}=\frac{1}{\omega_{b}} d x, \quad d p_{1}=\omega_{b} d p_{x} .
$$

According to the properties of the exterior product, the following equation holds:

$$
d q_{1} \wedge d p_{1}=d x \wedge d p_{x}
$$

The same can be done for all other rescalings. As a result, the Hamiltonian function becomes

$$
H=\frac{1}{2} \omega_{b}\left(p_{1}^{2}-q_{1}^{2}\right)+\frac{1}{2} \omega_{y}\left(p_{1}^{2}+q_{1}^{2}\right)+a \omega_{b}^{3} q_{1}^{3}+b \omega_{b} \omega_{y}^{2} q_{1} q_{2}^{2} .
$$


Expression (9) can be written in a much simpler form by introducing the coordinates

$$
\begin{gathered}
Q_{1}=\frac{1}{\sqrt{2}}\left(p_{1}+q_{1}\right), \quad P_{1}=\frac{1}{\sqrt{2}}\left(p_{1}-q_{1}\right), \\
Q_{2}=\frac{1}{\sqrt{2}}\left(q_{2}-i p_{2}\right), \quad P_{2}=\frac{1}{\sqrt{2}}\left(p_{2}-i q_{2}\right) .
\end{gathered}
$$

The same as before, these transformations are canonical and easy to verify

$$
\begin{gathered}
d P_{1}=\frac{-1}{\sqrt{2}} d q_{1}+\frac{1}{\sqrt{2}} d p_{1}, \quad d Q_{1}=\frac{1}{\sqrt{2}} d p_{1}+\frac{1}{\sqrt{2}} d q_{1} \\
d P_{1} \wedge d Q_{1}=\frac{-1}{2} d q_{1} \wedge d p_{1}+\frac{-1}{2} d q_{1} \wedge d q_{1}+\frac{1}{2} d p_{1} \wedge d p_{1}+\frac{1}{2} d p_{1} \wedge d q_{1}=d p_{1} \wedge d q_{1} .
\end{gathered}
$$

According to the normal form process (more details can be found in $[9,12,13]$ ), the Hamiltonian function is defined as follows

$$
H(Q, P)=H_{2}(Q, P)+\epsilon H_{3}(Q, P)+\ldots,
$$

thus

$$
H_{2}(Q, P)=\omega_{b} Q_{1} P_{1}+i \omega_{y} Q_{2} P_{2}
$$

Next, we calculate $H_{3}$ terms in the new coordinates $Q_{1}, Q_{2}, P_{1}, P_{2}$.

From the canonical transformation, we have

$$
q_{1}=\frac{1}{\sqrt{2}}\left(Q_{1}-P_{1}\right), \quad q_{2}=\frac{1}{\sqrt{2}}\left(Q_{2}+i P_{2}\right) .
$$

Hence

$$
\begin{aligned}
H_{3}= & \frac{a \omega_{b}^{3}}{2 \sqrt{2}}\left(Q_{1}^{3}-3 Q_{1}^{2} P_{1}+3 Q_{1} P_{1}^{2}-P_{1}^{3}\right) \\
& +\frac{b \omega_{b} \omega_{y}^{2}}{2 \sqrt{2}}\left(Q_{1} Q_{2}^{2}+2 i Q_{1} Q_{2} P_{2}-Q_{1} P_{2}^{2}-P_{1} Q_{2}^{2}-2 i P_{1} Q_{2} P_{2}+P_{1} P_{2}^{2}\right) .
\end{aligned}
$$

Now, the generating function $W_{3}$ of order three associated with $H_{3}$ is as follows:

$$
\begin{aligned}
W_{3}= & a_{1} Q_{1}^{3}+a_{2} Q_{1} Q_{2}^{2}+a_{3} P_{1}^{3}+a_{4} P_{1} P_{2}^{2}+a_{5} Q_{1}^{2} P_{1}+a_{6} Q_{1} P_{1}^{2} \\
& +a_{7} Q_{1} P_{2}^{2}+a_{8} P_{1} Q_{2}^{2}+a_{9} Q_{1} Q_{2} P_{2}+a_{10} P_{1} Q_{2} P_{2} .
\end{aligned}
$$

The coefficients $\left(a_{1}, \ldots, a_{10}\right)$ can be determined by setting

$$
H_{3}+\left\{W_{3}, H_{2}\right\}=0
$$

The coefficients have been calculated, and hence, we get

$$
\begin{aligned}
& a_{1}=\frac{-a \omega_{b}^{2}}{6 \sqrt{2}}, \quad a_{2}=\frac{-b \omega_{b} \omega_{y}^{2}}{2 \sqrt{2}\left(\omega_{b}+2 i \omega_{y}\right)}, \quad a_{3}=\frac{a \omega_{b}^{2}}{6 \sqrt{2}}, \\
& a_{4}=\frac{b \omega_{b} \omega_{y}^{2}}{2 \sqrt{2}\left(\omega_{b}+2 i \omega_{y}\right)}, \quad a_{5}=\frac{3 b \omega_{y}^{2}}{2 \sqrt{2}}, \quad a_{6}=\frac{3 b \omega_{b}}{2 \sqrt{2}}, \\
& a_{7}=\frac{b \omega_{b} \omega_{y}^{2}}{2 \sqrt{2}\left(\omega_{b}-2 i \omega_{y}\right)}, \quad a_{8}=\frac{b \omega_{b} \omega_{y}^{2}}{2 \sqrt{2}\left(2 i \omega_{y}-\omega_{b}\right)},
\end{aligned}
$$




$$
a_{9}=\frac{-i b \omega_{y}^{2}}{\sqrt{2}}, \quad a_{10}=\frac{i b \omega_{y}^{2}}{\sqrt{2}} .
$$

Thus, the normal form in our example is $H_{2}$ with respect to the generating function $W_{3}$, whose coefficients are presented above.

\section{Conclusions}

In this paper, we presented a general calculation of normal forms of Hamiltonian systems that is quadratic polynomial in the positions only. Previously, in Section 2, we provided the Hamiltonian normal forms theory that led to a reduced Hamiltonian number of degrees of freedom. More precisely, the generalization of Hamiltonian normal forms theory contains a very important step-the so-called generating function $W$. This function plays an important role in transforming the old Hamiltonian $H$ into an equivalent Hamiltonian $K$ up to an existing order of approximation and taking into account that the Poisson bracket of each term of the transformed Hamiltonian system $K$ and the generating function $W$ will vanish. This procedure guarantees that the transformed Hamiltonian system, especially in the first orders of the transformed system, provides interesting information due to the linear approximation around the equilibrium. An example was provided to illustrate the theory part.

Acknowledgments: The author is supported by Qassim University and the Ministry of Education of Saudi Arabia.

Conflicts of Interest: The authors declare no conflict of interest.

\section{References}

1. Arnold, V.I. Geometrical Methods in the Theory of Ordinary Differential Equations; Springer: Berlin, Germany, 1983.

2. Verhulst, F. Nonlinear Differential Equations and Dynamical Systems; Springer: Berlin, Germany, 2000.

3. Haller, G.; Wiggins, S. Geometry and chaos near resonant equilibria of 3-DOF Hamiltonian systems. Physica D 1996, 90, 319-365.

4. Cary, J.R. Lie transform perturbation theory for Hamiltonian systems. Phys. Rep. 1981, 79, 129-159.

5. Deprit, A. Canonical transformations depending on a small parameter. Celest. Mech. 1969, 1, 12-30.

6. Meyer, K.R. Normal forms for Hamiltonian systems. Celest. Mech. 1974, 9, 517-522.

7. Meyer, K.R. Normal forms for the general equilibrium. Funkcialaj Ekvacioj 1984, 9, 261-271.

8. Henrard, J. On a perturbation theory using Lie transforms. Celest. Mech. 1970, 3, 107-120.

9. Palacian, J.; Yanguas, P. Reduction of polynomial Hamiltonians by the construction of formal integrals. Nonlinearity 2000, 13, p1021.

10. Van der Aa, E.; Verhulst, F. Asymptotic integrability and periodic solutions of a Hamiltonian system in 1:2:2 resonance. SIAM J. Math. Anal. 1984, 15, 890-911.

11. Ferrer, S.; Hanssmann, H.; Palacian, J.; Yanguas, P. On perturbed oscillators in 1:1:1 resonance: The case of axially symmetric cubic potentials. J. Geom. Phys. 2002, 40, 320-369.

12. Meyer, K.R.; Offin, D.C. Introduction to Hamiltonian Dynamical Systems and the N-Body Problem; Springer: Berlin, Germany, 1992.

13. Uzer, T.; Jaff, C.; Palacian, J.; Yanguas, P.; Wiggins, S. The geometry of reaction dynamics. Nonlinearity 2002, 15,4 .

14. Murdock, J. Normal forms and unfoldings for local dynamical systems. In Springer Monographs in Mathematics; Springer: New York, NY, USA, 2003.

15. Jorba, A. A Methodology for the Numerical Computation of Normal Forms, Centre Manifolds and First Integrals of Hamiltonian Systems. Exp. Math. 1999, 8, 155-195. 
16. Wiggins, S. Introduction to Applied Nonlinear Dynamical Systems and Chaos. In Texts in Applied Mathematics; Springer: Berlin, Germany, 1990; Volume 2.

17. Mikram, J.; Zinoun, F. Computation of normal forms of Hamiltonian systems in the presence of Poisson commuting integrals. Numer. Algorithms 1999, 21, 287-310.

18. Sanders, J.A.; Verhulst, F. Averaging Methods in Nonlinear Dynamical Systems. In Applied Mathematical Sciences; Springer: Berlin, Germany, 1985; Volume 59.

(C) 2017 by the authors. Licensee MDPI, Basel, Switzerland. This article is an open access article distributed under the terms and conditions of the Creative Commons Attribution (CC BY) license (http:/ / creativecommons.org/licenses/by/4.0/). 